\title{
LOS EFECTOS DE LA IMAGINERÍA MOTORA EN EL CONTROL POSTURAL: UN ESTUDIO LONGITUDINAL EN SUJETOS SANOS
}

\author{
Belén Zamarro-Rodríguez, MSc ${ }^{1}$; César Cuesta-García, $\mathrm{PhD}^{1,2}$; Sergio Lerma-Lara, $\mathrm{PhD}^{3}$. \\ 1. Grupo de Investigación Occupational Thinks. Centro Superior de Estudios Universitarios La Salle, Universidad Autónoma de Madrid, Madrid, España. \\ 2. Departamento de Terapia Ocupacional. Centro Superior de Estudios Universitarios La Salle, Universidad Autónoma de Madrid, Madrid. España. \\ 3. NeuroMechanics and Rehabilitarion Research Group. Departamento de Fisioterapia, Facultad de Ciencias de la Salud. Centro Superior de Estudios \\ Universitarios La Salle, Universidad Autónoma de Madrid, Madrid, España.
}

\section{Correspondencia:}

Belén Zamarro Rodríguez, OT. Facultad de Ciencias de la Salud. CSEU La Salle. Universidad Autónoma of Madrid. Calle La Salle, $n^{\circ}$ 10, 28023 Madrid, España Teléfono: (+34) 917401980 Fax: (+34) 913571730 E-Mail: bzamro@campuslasalle.es

\section{Conflicto de Intereses:}

Los autores del presente manuscrito declaran no presentar ningún conflicto de interés y además el presente estudio no ha sido presentado en ningún evento científico.

\section{Financiación}

Los autores declaran no haber recibido financiación/compensación para el desarrollo de esta investigación.

\section{Comité de Ética:}

CSEULS-PI-56/2015.

DOI:

https://doi.org/10.37382/jomts.v1i1.6

\section{Recepción del Manuscrito: \\ 24-Septiembre-2018}

\section{Aceptación del Manuscrito:}

2- Enero-2019

Licensed under: CC BY-NC-SA 4.0

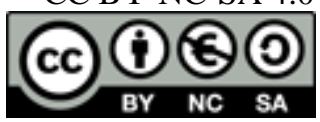

\section{RESUMEN}

Objetivo: Determinar los efectos de la imaginería motora sobre el control postural en bipedestación, postural de partida de una gran variedad de ocupaciones humanas.

Diseño: Estudio piloto experimental, de corte longitudinal.

Marco: Ámbito universitario

Participantes: Quince sujetos sanos de ambos sexos, con edades comprendidas entre los 18-30 años.

Intervenciones: Fueron evaluados en bipedestación sobre una plataforma dinamométrica computarizada (Neurocom) mediante el test de organización sensorial. Mientras se realizaban las pruebas, debían imaginarse dos situaciones: 1) Una situación de estabilidad, poniendo el foco atencional en el feedback intrínseco y 2) Otra situación dinámica, poniendo el foco atencional en el feedback extrínseco. Un relato auditivo, acompañado de efectos sonoros, fue añadido al procedimiento de imaginería motora.

Variables: Puntuaciones en el equilibrio, estrategias somatosensoriales, desplazamiento del centro de gravedad, el perfil sensorial.

Resultados: El análisis reveló diferencias significativas $(p<0,01)$ en las variables cuando los participantes imaginaban la situación de estabilidad. Se comprobó una relación entre dichos resultados y la puntuación del perfil sensorial.

Conclusión: La imaginería motora, es un procedimiento que ha demostrado influencias en la mejora del control postural en bipedestación y puede ser una técnica que complemente el tratamiento de pacientes con alteraciones posturales y del equilibrio.

Palabras Clave: Equilibrio postural, Centro de gravedad, Imaginería motora, Posturografía. 


\section{INTRODUCCIÓN}

El control postural establece las bases para una buena calidad de vida y por consiguiente el desarrollo óptimo de nuestras ocupaciones, ya que la postura se relaciona directamente con el estado de salud de la persona.

La Terapia Ocupacional es una disciplina sociosanitaria encargada de la intervención en las diferentes áreas de la ocupación, concretamente trabajando con aquellas personas quienes tienden a encontrarse ante una situación de disfunción ocupacional, impidiéndoles el desarrollo de unos hábitos de vida normales.

Para el desarrollo del desempeño ocupacional es necesaria la realización de patrones de ejecución, donde la estabilidad postural en bipedestación resulta ser una condición indispensable.

La realización de las actividades cotidianas requiere un control flexible de la postura, incluso en las actividades relativamente sencillas como es ponerse un jersey, se precisan complejas interacciones entre los diferentes sistemas que intervienen en el control del movimiento y la postura.

Dicha estabilidad es alcanzada a través de estructuras funcionales como la corteza motora primaria, corteza visual, corteza somatosensorial, parte anterior y posterior del cerebelo, ganglios basales (sobre todo el putamen) y el tronco cerebral (Moya et al., 2005; Taube et al., 2015).

Las estrategias de equilibrio incluyen adaptaciones visuales, vestibulares y somatosensoriales que desencadenan tres tipos de respuestas o estrategias motoras: las primeras ocurren a nivel del tobillo; en segundo lugar, aparecen las estrategias de cadera y en último lugar o en caso de necesidad, la estrategia de paso, incluyendo múltiples articulaciones actuando en conjunto (Shumway Cook, 1995).

Aunque es escaso el número de investigaciones realizadas, se ha podido observar una influencia positiva por parte de la imaginería motora (IM) a la hora del mantenimiento del control postural, debido a que ésta comparte casi el mismo sustrato neurobiológico que las percepciones sensoriales.

Stephen Kosslyn la define como «la presencia de una representación mental mientras el estímulo no está siendo percibido, esta fue creada durante la fase de percepción, tiene las características del estímulo y dan la experiencia subjetiva de percepción» (Tamayo Martínez, 2014).

Con relación al análisis del equilibrio, una de las herramientas utilizadas es la posturografía dinámica computarizado, que estudia el control postural a través de los movimientos del centro de presión sobre una plataforma dinamométrica.

Dentro de las posibles opciones de evaluación, encontramos el test de organización sensorial (TOS), por medio del cual es posible determinar la aportación de cada sistema (visual, vestibular y propioceptivo), en seis condiciones diferentes (Rama López \& Pérez Fernández, 2003).

Tras ello, la hipótesis del estudio planteado es la de demostrar que la IM influye en el control postural estático en la bipedestación de sujetos sanos. El objetivo principal fue analizar la bipedestación estática pre y post intervención con IM.

Para ello, se plantearon los siguientes objetivos específicos: a) analizar y comparar los cambios que se producen en las puntuaciones de equilibrio, estrategias motoras y desplazamiento del centro de gravedad en una situación basal y una situación posterior a diferentes intervenciones con IM y b) determinar la influencia de estas intervenciones en los sistemas vestibular y somatosensorial de los participantes del estudio.

Como objetivo secundario se determina la relación existente entre el perfil sensorial de los sujetos analizados, identificado por su desempeño ante determinadas ocupaciones, así como las diferentes intervenciones de IM realizadas. 


\section{MÉTODOS}

\section{Diseño de estudio}

Se realizó un estudio piloto longitudinal de tipo panel, presentando una evaluación en dos tiempos: primero, tiempo 1 como situación control, dónde no se realizó ninguna intervención y tiempo 2 , donde tuvo lugar el desarrollo de la intervención con IM.

Dicho estudio de observación es un estudio de investigación básica desarrollada en el Instituto de Rehabilitación Funcional La Salle (Madrid) durante los meses de marzo y abril del año 2016, siendo éste un espacio cerrado y controlado por el investigador, quien de primera mano recogió todos los datos a través de las diferentes técnicas de valoración.

\section{Participantes}

Los criterios de inclusión para participar en el estudio fueron los siguientes: sujetos sanos, de ambos sexos, que no presentaran ninguna patología diagnosticada, ni deficiencias sensoriales, con edades comprendidas entre los 18-30 años. Se excluyeron todos aquellos sujetos que presentaran cualquier tipo de patología músculo-esquelética y dolor en el momento actual. Los sujetos de la muestra fueron seleccionados al azar a través de un muestreo no probabilístico por bola de nieve.

\section{Medidas de resultado}

Las herramientas utilizadas fueron:

- Plataforma dinámica computarizada (Plataforma Smart-Equitest de Neurocom ${ }^{\circledR}$ ), en concreto el test de organización sensorial (TOS), cuyos datos reflejan la puntuación total en el equilibrio, el tipo de estrategias somatosensoriales (de tobillo, cadera y paso) y el rango de desplazamiento del centro de gravedad.

\section{- Sensory Profile Adults}

El cuestionario autoadministrado "Sensory Profile Adults de Catana Brown y Winnie Dunn".

\section{Procedimientos}

Los procedimientos seguidos en la investigación se han realizado conforme a las normas del Comité Ético de Investigación del Centro Superior de Estudios
Universitarios La Salle con el siguiente número de registro CSEULS-PI-56/2015.

El estudio comenzó con la administración de un consentimiento informado y la cumplimentación del Sensory Profile Adults de Catana Brown y Winnie Dunn (2002) donde debían cuantificar a través de los calificadores nunca, casi nunca, frecuentemente, siempre y casi siempre, diferentes situaciones sensoriales de su vida diaria.

Tras ello se pasó a la administración de la prueba de Posturografía en bipedestación, con la plataforma dinámica computarizado, en la cual, se coloca al sujeto con un arnés y éste pasa a ser evaluado en 6 condiciones sensoriales diferentes, viéndose así el trabajo de los diferentes sistemas somatosensoriales implicados (Figura 1).

Para el tiempo 1, se administró el TOS, donde sólo se analizaron los resultados de la condición 2 (ojos cerrados, plataforma fija) y 5 (ojos cerrados, plataforma móvil). Se eligieron ambas condiciones por ser las únicas dónde se elimina el input visual; de esta manera garantizamos el procedimiento de IM y se analiza específicamente el funcionamiento del sistema vestibular y somatosensorial. En este primer tiempo, fue recogida la situación basal de los participantes del estudio en bipedestación, sin llevarse a cabo ningún tipo de intervención. Pasadas dos horas, se realizó la intervención de IM, que incorporaba un feedback auditivo de 90 segundos para cada una de las condiciones.

En la condición 2 (ojos cerrados sobre una plataforma fija), durante el momento de la intervención, los sujetos debían imaginarse en una montaña rusa. El relato del investigador que narraba la situación se acompañó de un audio que reproducía con exactitud los sonidos de la montaña rusa, haciendo que el protagonismo de lo vivenciado recayera en un feedback extrínseco.

En la condición 5 (ojos cerrados sobre una plataforma móvil) se inducían a la imaginación de una ducha relajante. En este caso, un relato auditivo, les 
Figura 1. Prueba del Test de organización sensorial (TOS), posturografía dinámica computarizada Neurocom.

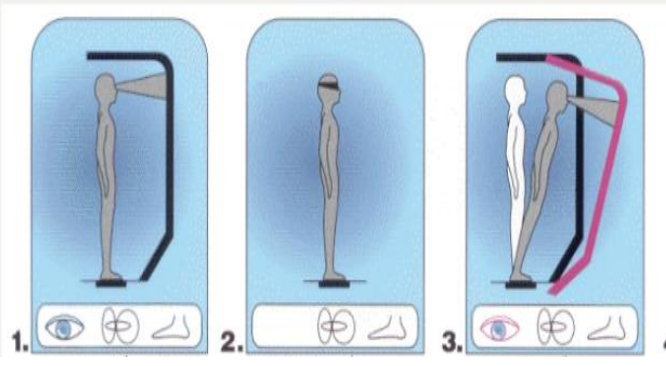

hacía tomar consciencia de su esquema corporal, recayendo el foco atencional hacia un feedback intrínseco, así como su sistema somatosensorial. Durante estas intervenciones, se realizó la segunda valoración (tiempo 2).

Al tratarse de un estudio piloto, encontramos sesgos durante el proceso de selección del muestreo, no llegando a ser una muestra representativa para la Comunidad de Madrid. De esta misma manera, contamos también con sesgos de concepto, al no considerar variables como el factor aprendizaje que pueden afectar a los resultados obtenidos.

\section{Análisis estadístico}

Finalmente, el análisis estadístico se llevó a cabo con el sistema SPSS versión 20.0. Pasadas las pruebas de normalidad de Shapiro-Wilk, tuvo lugar la prueba de los Rangos con signo de Wilcoxon (aportando un intervalo de confianza del 95\%). Se estableció un análisis de correlaciones bivariadas entre los resultados obtenidos durante la intervención y el Sensory Profile Adults, a través del estadístico de Spearman y la significancia estadística del mismo, aportando si la relevancia es significativa al 0,05 o al 0,01 .

\section{RESULTADOS}

Fueron evaluados quince sujetos $(73,3 \%$ mujeres y $26,7 \%$ hombres), con una media de edad de $22,27 \pm 1,83$ años.

La muestra se dividió en: Grupo 1, con edades entre 18-22 años, y Grupo 2, con edades entre 23-30 años. Todos ellos fueron sujetos sin ningún tipo de
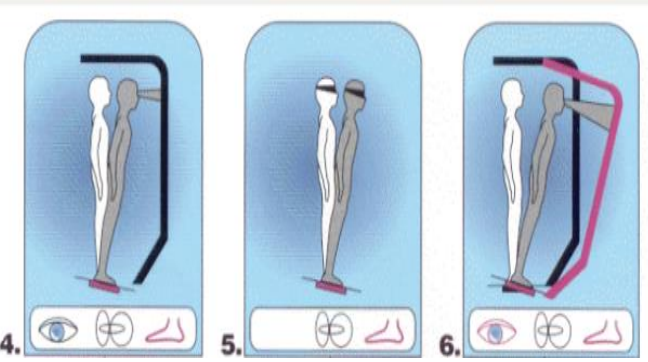

patología, los cuales formaban parte del ámbito universitario y residentes en la Comunidad de Madrid, no realizaban deporte de manera regular y su IMC alcanzaba una media de $23,89 \pm 4,07 \mathrm{~kg} / \mathrm{m}^{2}$.

Los resultados del test Sensory Profile Adults mostraron en Registro Bajo, para el 46,7\% de la muestra una puntuación superior a la norma. Sin embargo, en Búsqueda de Sensaciones (73,3\%), Sensibilidad Sensorial (66,7\%) y Evitación de sensaciones $(60 \%)$ presentaron puntuaciones típicas.

En el Grupo 1 alcanzan principalmente puntuaciones acordes a la normalidad (Figura 2), mientras que en el Grupo 2 encontramos puntuaciones superiores a las típicas, para los ítems de Registro Bajo, así como Evitación de las Sensaciones (Figura 3). Tras el análisis sensorial realizado a través del TOS, los participantes mostraron peores resultados relacionados con el sistema vestibular.

Figura 2: Gráfico de barras sobre los resultados obtenidos en el Perfil Sensorial previo a la intervención en el Grupo 1 (18-22 años).

\section{Perfil Sensorial (Grupo 1)}

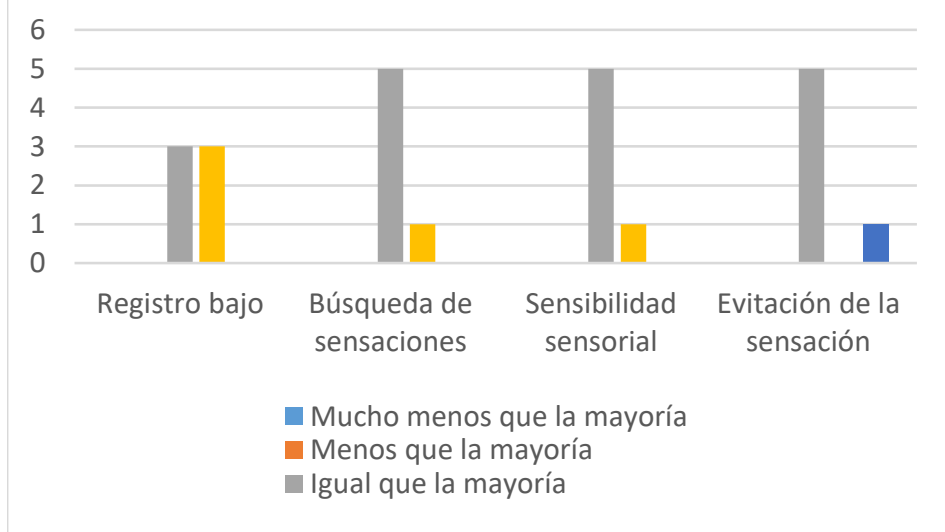


Figura 3. Gráfico de barras sobre los resultados obtenidos en el Perfil Sensorial previo a la intervención en el Grupo 2 (23-30 años).

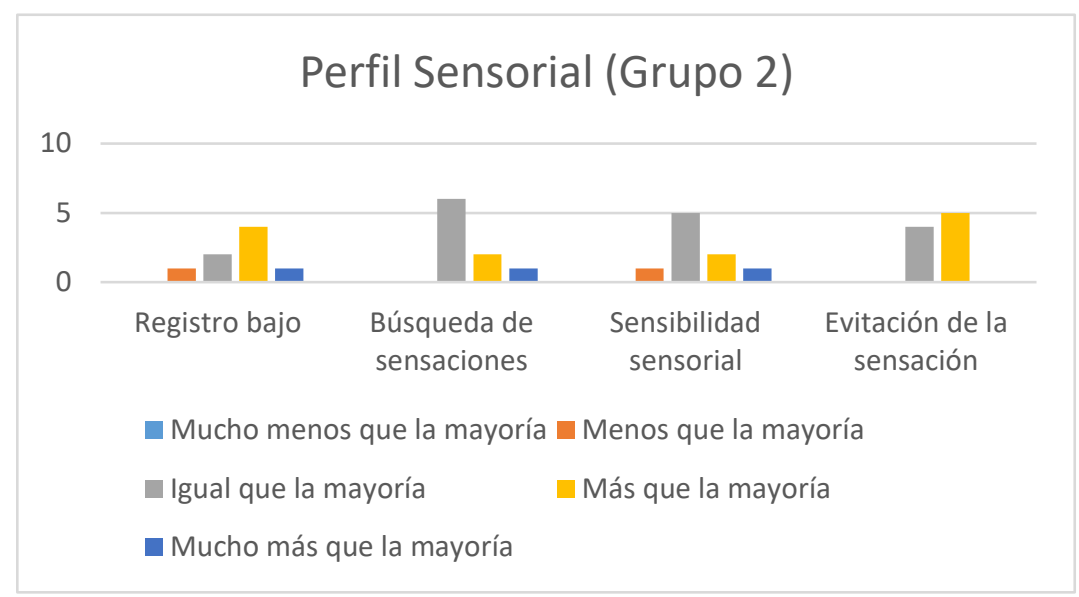

Cuatro de los sujetos, obtuvieron puntuaciones comprendidas entre el $25-50 \%$ y seis con una puntuación entre el 50-75\%. Estos resultados fueron más negativos a medida que aumentaba la edad.

Para las puntuaciones de las variables de equilibrio, estrategias motoras y desplazamiento del centro de gravedad en la condición cinco, previa a la intervención, fueron de nuevo los participantes del Grupo 2, quienes mostraron mejor equilibrio, mientras que, para la segunda condición, las puntuaciones fueron prácticamente similares.

Tras la realización de la prueba de rangos con signo de Wilcoxon, observamos que, a pesar de las consignas de inestabilidad ofertadas durante la segunda condición, no hubo cambios estadísticamente significativos; no obstante, las puntuaciones en el equilibrio disminuyeron 0,79 puntos, presentando un menor número de estrategias de equilibrio y un desplazamiento del centro de gravedad significativo (pvalor $=0,009$. Z -2,613).

Por otro lado, un gran número de sujetos pertenecientes al Grupo 2, referían sentir ansiedad durante el proceso de intervención con IM para esta segunda condición.

En la condición cinco, la realización de la intervención supuso un mayor control postural para cada uno de los participantes, adquiriendo valores estadísticamente significativos en todas las variables (Tabla 1).

Tabla 1. Diferencias entre las puntuaciones obtenidas en las distintas variables analizadas a través de la posturografía tras la aplicación de imaginería motora para las condiciones 2 (ojos cerrados plataforma fija) y 5 (ojos cerrados plataforma móvil) del Test de Organización Sensorial (Neurocom).

\begin{tabular}{|c|c|c|c|c|}
\hline & Condición & Pre-Intervención & Post-Intervención & $\begin{array}{c}\text { Diferencia de cambio } \\
\text { dentro del grupo }\end{array}$ \\
\hline \multirow[t]{2}{*}{ Equilibrio } & 2 & $92,79 \pm 2,29$ & $92,46 \pm 2,68$ & ,33 (-5,34 a 6,00) \\
\hline & 5 & $61,99 \pm 14,94$ & $74,08 \pm 8,68$ & $-12,09(-40,34$ a 8,66$)$ \\
\hline \multirow[t]{2}{*}{ Estrategias } & 2 & $96,88 \pm 1,43$ & $94,77 \pm 6,90$ & $2,10(-4,34$ a 28,33$)$ \\
\hline & 5 & $79,32 \pm 7,83$ & $85,13 \pm 5,92$ & $-5,80^{*}(-25,67$ a 7,00$)$ \\
\hline \multirow{2}{*}{$\begin{array}{l}\text { Desplazamiento } \\
\text { del centro de } \\
\text { gravedad }\end{array}$} & 2 & ,62, \pm 59 & ,92, \pm 68 &,$- 29(-2,00$ a 1,47$)$ \\
\hline & 5 & ,78, \pm 63 &, $75 \pm, 49$ &, $026^{*}(-, 97$ a 1,73$)$ \\
\hline
\end{tabular}


De esta manera, tras el desarrollo de la intervención, tuvo lugar un incremento en sus puntuaciones en el equilibrio, viéndose ésta aumentada en 13,34 puntos y disminuyendo, por tanto, el desplazamiento de su centro de gravedad en 0,58 puntos.

Tras el desarrollo de dicho análisis, pasamos a determinar las correlaciones existentes entre los resultados obtenidos con el empleo de la intervención (IM) y la administración del Perfil Sensorial.

Las correlaciones más fuertes vienen dadas por los ítems correspondientes al procesamiento visual, nivel de actividad, procesamiento del movimiento $\mathrm{y}$ procesamiento auditivo (Tabla 2).

Es en el Grupo 2, según el Perfil Sensorial, quienes al imaginarse en una situación de total desequilibrio refirieron abrumarse en sitios llenos de gente y tras gran cantidad de movimiento a su alrededor, dificultades de concentración e incluso miedo a las alturas.

Mientras tanto, los mejores resultados encontrados en la condición cinco tras imaginarse en una situación de total estabilidad, fueron presentados por quienes refirieron tener una peor capacidad de concentración.

\section{DISCUSIÓN}

La IM genera cambios significativos en el control postural. Para Hamel y Lajoie, y años posteriores Tia (Mouthon et al., 2015), tiene lugar una activación corticoespinal además de un aumento del flujo sanguíneo en las áreas motoras, los cuales podrían estar relacionados con los resultados presentados.

Tabla 2. Correlaciones de Spearman entre las puntuaciones obtenidas a través del sistema de posturografía tras la intervención y el Perfil Sensorial de cada uno de los participantes del estudio. Aportando valores de significancia del 0,05 y 0,01.

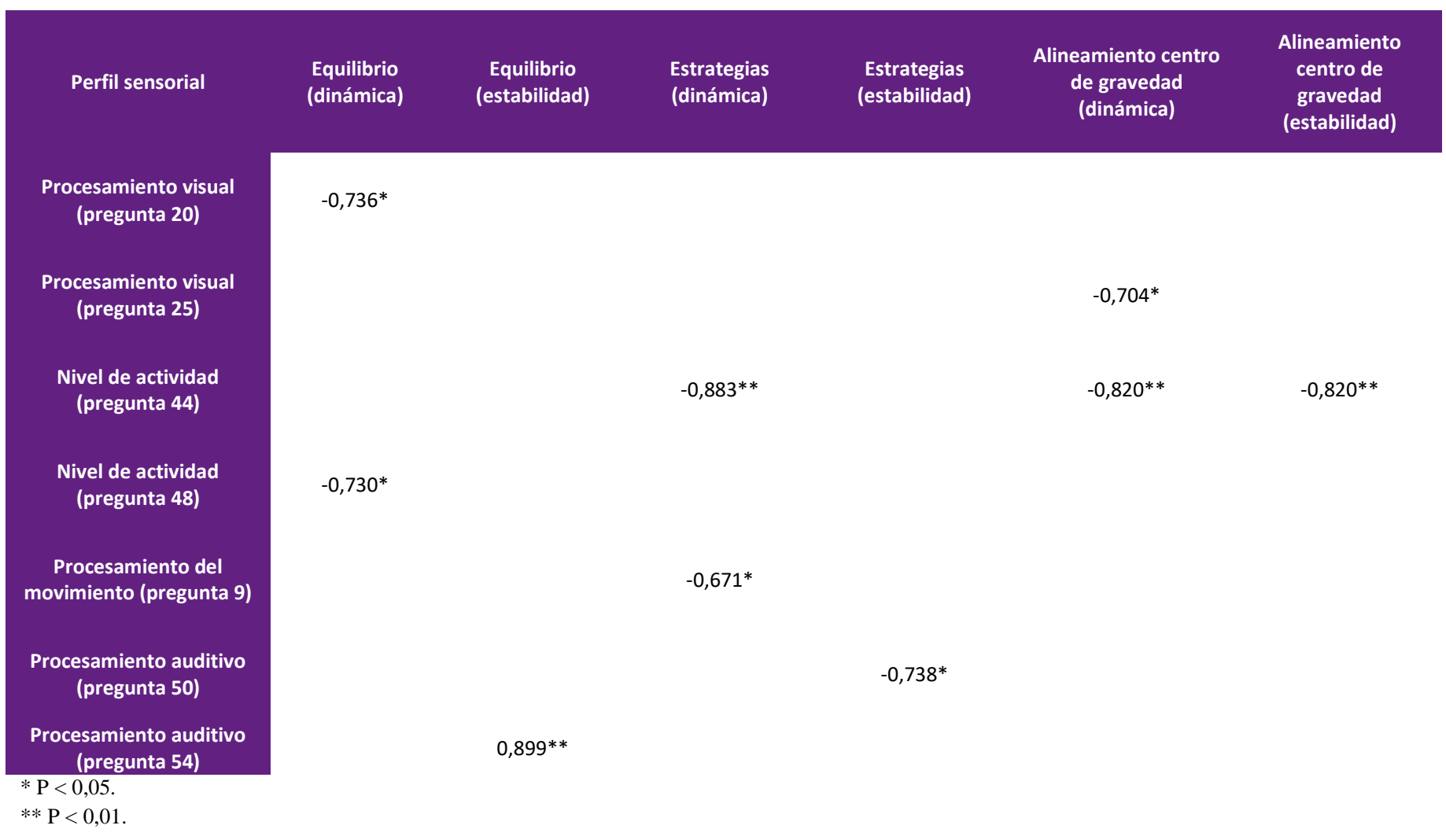


Durante el desarrollo de la intervención, tanto en la segunda como quinta condición, tiene lugar un cambio en el equilibrio. La IM ha contribuido a la facilitación del acto motor, produciéndose según otras evidencias científicas, las activaciones de las regiones neuronales comunes a tal acto (Carvalho Rodrigues et al., 2003).

Al imaginarse y vivenciar el movimiento en primera persona, aumentó la integración de la información propioceptiva, que contribuyó a activar el sistema postural. El input auditivo proporcionado en el estudio, permitió dicha representación mental (Oostra et al., 2015).

En la condición dos, al realizar la intervención, observamos una mayor inestabilidad postural, generando una mayor frecuencia de estrategias de cadera, con el objetivo de poder restablecer su centro de gravedad y generar los ajustes posturales necesarios (Calderón \& César Legido, 2002).

Otro hallazgo encontrado, ante la IM de una situación inestable, fue el aumento en el desplazamiento del centro de gravedad (Rodrigues et al., 2010; Hanakawa, 2015). Estudios recientes corroboran tales resultados, sin embargo, esta influencia se produce sólo en los participantes con altos niveles de intensidad del movimiento imaginado (Lemos et al., 2014; Souza et al., 2015).

Además, los sujetos debieron centrar su atención hacia un foco atencional externo, el cual puede haber aumentado su excitación. Nos encontramos con una posible relación entre las estrategias de excitación y el equilibrio postural, aunque en la actualidad no hay investigaciones profundas sobre el tema (Andersson et al., 2002).

El presente estudio incluyó la ejecución combinada de una tarea postural (bipedestación estática) y una tarea cognitiva (imaginería motora). La literatura sugiere que el mantenimiento del equilibrio estático es en gran parte automatizado, por lo tanto, requiere de unos recursos de atención sensibles a la actividad cognitiva.
La incapacidad que el sujeto tiene para asignar suficiente atención al mantenimiento postural durante la realización de multitareas es un factor que contribuye al desequilibrio (Shumway-Cook \& Woollacott, 2000; Stins et al., 2015).

Para aquellas condiciones sensoriales de mayor dificultad, el añadir una segunda tarea facilita la pérdida de equilibrio en todas aquellas personas que presentan antecedentes de inestabilidad, ya que el tiempo de reacción empleado para ajustarse al cambio postural, se ve incrementado.

Según nuestros resultados, el grupo de edades superiores, mostraron peores puntuaciones en el equilibrio. Este grupo, además, presentaba dificultades en la atención mantenida. Sin embargo, al imaginarse una situación de completa estabilidad, presentaron una gran mejoría en su control postural. Esto hace que produzcan cambios en el registro sensorial exteroceptivo, consiguiendo una mayor concienciación de su sistema propioceptivo (Aguilar Rebolledo, 2006).

Como podemos ver y corroborado con anterioridad (Antonietti, 2009), a medida que se incrementa la edad, este proceso en el que es necesario cumplir ciertas exigencias atencionales producidas por esa doble tarea, resulta ser mucho más costoso, ya que con el paso de los años nuestros tiempos de reacción resultan ser más prolongados.

En nuestro experimento, los relatos auditivos, van acompañados de melodías específicas. Como ya observaron Antic et al. (2008), el uso de la música resulta ser una buena técnica para la prevención de caídas o vértigos, ya que puede proporcionar los ritmos secuenciales necesarios para llevar a cabo las acciones motoras.

Con relación a las estrategias llevadas a cabo en esa condición, se observó una tendencia a realizar estrategias de tobillo, tratando de mantener la verticalidad a través de la alineación de su postura. 
Cuando la cantidad de atención invertida en el control postural se reduce mediante la introducción de una tarea cognitiva cargada de información propioceptiva, podemos observar que las fluctuaciones que tienen lugar tras el desplazamiento del centro de gravedad alcanzan valores similares a los observados durante la situación basal (sujetos en bipedestación sobre un soporte móvil, privados del input visual).

Este hallazgo sugiere que como comentaron Hunter y Hoffman (Donker et al., 2007) al estar de pie privados del input visual, los sujetos sienten una mayor necesidad de llevar a cabo una conciencia postural, por lo que imaginarse en una situación de estabilidad facilita el trabajo postural de manera automática y eficaz.

Durante el desarrollo de dicho proyecto, tuvieron lugar una serie de sesgos, principalmente sesgos de concepto al no considerar la presencia de un factor aprendizaje tras el pequeño periodo dado entre ambas intervenciones. De la misma manera, encontramos un factor emocional el cual no llegó a ser contemplado, pudiendo influir en los resultados obtenidos.

Sería interesante poder llevar a cabo un entrenamiento previo en el uso de la imaginería, ya que el tamaño del efecto podría ser mayor en las variables estudiadas, así como la administración de cuestionarios con los que fuera posible determinar la capacidad de representación mental de cada uno de ellos.

Por otro lado, contamos con un tamaño muestral pequeño por lo que genera una falta de representatividad de la muestra estudiada, de esta manera no es posible la extrapolación de los datos a la Comunidad de Madrid ya que además son sujetos evaluados en una zona concreta de la comunidad.

Aunque el estudio ha presentado ciertas limitaciones, tras la mejoría obtenida en la condición cinco, hace plantearnos la posibilidad de continuar la investigación con participantes que presenten alteraciones del equilibrio, viendo así si se produce un mantenimiento en los resultados alcanzados. A pesar de todo, seguimos encontrándonos con poca literatura la cual pueda explicar con mayor detalle, los datos obtenidos tras la realización del estudio.

\section{CONCLUSIÓN}

El empleo de la IM acompañada de información auditiva trae consigo cambios en el control postural de las personas jóvenes.

El proceso de imaginación de una situación estable en bipedestación permite adquirir una mayor conciencia corporal y mejoras en el control postural y equilibrio.

Esto supone, una futura vía de intervención complementaria al tratamiento, ya que las mejoras alcanzadas a nivel postural permiten la mejor realización del desempeño de todas aquellas actividades de la vida diaria donde se ve implícito el mantenimiento de una buena postura para su desarrollo óptimo. Por consiguiente, es posible verse mejoras en sus ocupaciones.

Tras la accesibilidad de la técnica y los resultados positivos en sujetos sanos, consideramos oportuno continuar investigando sobre su aplicación en personas con alteraciones del equilibrio. $\mathrm{Y}$ contrastar la influencia de dicha intervención en el desarrollo ocupacional de las personas.

\section{FRASES DESTACADAS}

$>$ La imaginería motora factor influyente en el control postural de las personas sanas.

$>$ La toma de conciencia corporal influyente en el equilibrio postural.

$>$ La representación mental de situaciones estables genera mejoras en el equilibrio.

$>$ La estabilidad postural es una condición importante en las ocupaciones. 


\section{REFERENCIAS}

Aguilar Rebolledo F. La musicoterapia como instrumento favorecedor de la plasticidad, el aprendizaje y la reorganización neurológica. Plast Rest Neurol. 2006;5(1):8597.

Andersson G, Hagman J, Talianzadeh R, Svedberg A, Larsen HC. Effect of cognitive load on postural control. Brain Res Bull. 2002;58(1):135-9 DOI: http://dx.doi.org/10.1016/S03619230(02)00770-0.

Antic S, Galinovic I, Lovrendic-Huzjan A, Vukovic V, Jurasic M-J, Demarin V. Music as an auditory stimulus in stroke patients. Coll Antropol. 2008;32:19-23.

Antonietti A. Why is music effective in rehabilitation? Stud Health Technol Inform. 2009;145:179-94 DOI: http://dx.doi.org/10.3233/978-1-60750-018-6-179.

Calderón F, César Legido AJ. Neurofisiología aplicada al deporte. 2002.

Carvalho Rodrigues E, Imbiriba LA, Rego Leite G, Magalhães J, Volchan E, Vargas CD. Mental stimulation strategy affects postural control. Rev Bras Psiquiatr. 2003;25:33-5 DOI: http://dx.doi.org/S1516-44462003000600008 [pii].

Donker SF, Roerdink M, Greven AJ, Beek PJ. Regularity of centerof-pressure trajectories depends on the amount of attention invested in postural control. Exp Brain Res. 2007;181(1):111 DOI: http://dx.doi.org/10.1007/s00221-007-0905-4.

Hanakawa T. Organizing motor imageries. Neurosci Res. 2015;104:56-63.

Lemos T, Souza NS, Horsczaruk CHR, Nogueira-Campos A a, de Oliveira L a S, Vargas CD, Rodrigues EC. Motor imagery modulation of body sway is task-dependent and relies on imagery ability. Front Hum Neurosci. 2014;8:290 DOI: http://dx.doi.org/10.3389/fnhum.2014.00290.

Mouthon A, Ruffieux J, Waelchli M, Keller M, Taube W. TaskDependent Changes of Corticospinal Excitability During Observation and Motor Imagery of Balance Tasks. Neuroscience. $\quad 2015 ; 303(\mathrm{Mi}): 535-43 \quad$ DOI: http://dx.doi.org/10.1016/j.neuroscience.2015.07.031.
Moya MFPDE, Bertomeu JMB, Vivas MJ, Biomecánica I De, Universidad DV, Valencia P De. Evaluación y rehabilitación del equilibrio mediante posturografía. 2005;39(6):315-23.

Oostra KM, Oomen A, Vanderstraeten G, Vingerhoets G. Influence of motor imagery training on gait rehabilitation in sub-acute stroke: A randomized controlled trial. J Rehabil Med. 2015;47(3):204-9 DOI: http://dx.doi.org/10.2340/165019771908.

Rama López J, Pérez Fernández N. Pruebas vestibulares y posturografía. Rev Med Univ Navarra. 2003;47(4):21-8.

Rodrigues EC, Lemos T, Gouvea B, Volchan E, Imbiriba LA, Vargas CD. Kinesthetic motor imagery modulates body sway. Neuroscience. Elsevier Inc.; 2010;169(2):743-50 DOI: http://dx.doi.org/10.1016/j.neuroscience.2010.04.081.

Shumway Cook A. Control Motor: Teoría y aplicaciones prácticas. Washington; 1995.

Shumway-Cook A, Woollacott M. Attentional demands and postural control: the effect of sensory context. J Gerontol A Biol Sci Med Sci. 2000;55(1):10-6 DOI: http://dx.doi.org/10.1093/gerona/55.1.M10.

Souza NS, Carolina A, Martins G, Canuto S. Postural control modulation during motor imagery tasks: a systematic review. Int Arch Med. 2015;8(2):1-12 DOI: http://dx.doi.org/10.3823/1642.

Stins J, Schneider IK, Stins JF, Schneider IK, Koole SL, Beek PJ. The Influence of Motor Imagery on Postural Sway: Differential Effects of Type of Body Movement and Person Perspective Th e In fl uence of Motor Imagery on Postural Sway: Di ff erential E ff ects of Type of Body Movement and Person Perspective. Adv Cogn Psychol. 2015;11(April 2016):77-83 DOI: http://dx.doi.org/10.5709/acp-0173-x.

Tamayo Martínez N. Imaginería mental: neurofisiología e implicaciones en psiquiatría. Rev Colomb. 2014;43(1):40-6.

Taube W, Mouthon M, Leukel C, Hoogewoud HM, Annoni JM, Keller M. Brain activity during observation and motor imagery of different balance tasks: An fMRI study. Cortex. 2015;64:102-14 DOI: http://dx.doi.org/10.1016/j.cortex.2014.09.022. 